An Interior-point Method with Polynomial

Complexity and Superlinear Convergence for Linear Complementarity Problems

Jun Ji

F. Potra

R.A. Tapia

Y. Zhang

July, 1991

TR91-23 



\title{
An Interior-Point Method with Polynomial Complexity and Superlinear Convergence for Linear Complementarity Problems
}

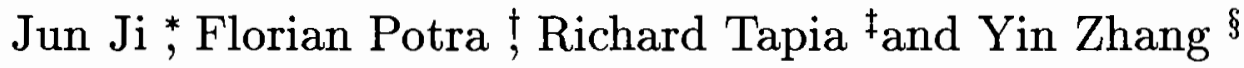 \\ July, 1991
}

Key Words: Linear programming, quadratic programming, linear complementarity problems, primal-dual interior-point algorithms, polynomiality, superlinear convergence.

Abbreviated Title: Interior-Point Methods for Linear Complementarity Problems

\footnotetext{
* Department of Mathematics, The University of Iowa, Iowa City, Iowa 52242

${ }^{\dagger}$ Department of Mathematics, The University of Iowa, Iowa City, Iowa 52242 and visiting member of the Center for Research on Parallel Computation, Rice University, Houston, Texas, 77251-1892. Research supported in part by NSF Coop. Agr. No. CCR-8809615.

${ }^{\ddagger}$ Department of Mathematical Sciences and the Center for Research on Parallel Computation, Rice University, Houston, Texas, 77251-1892. Research supported in part by NSF Coop. Agr. No. CCR8809615, AFOSR 89-0363, DOE DEFG05-86ER25017 and ARO 9DAAL03-90-G-0093.

$\S$ Department of Mathematics and Statistics, University of Maryland Baltimore County, Baltimore, MD 21228 and visiting member of the Center for Research on Parallel Computation, Rice University, Houston, Texas, 77251-1892. Research supported in part by NSF Grant DMS-9102761 and NSF Coop. Agr. No. CCR-8809615.
} 



\begin{abstract}
For linear programming, a primal-dual interior-point algorithm was recently constructed by Zhang and Tapia that achieves both polynomial complexity and $Q$-superlinear convergence ( $Q$-quadratic in the nondegenerate case). In this paper, we extend their results to quadratic programming and linear complementarity problems.
\end{abstract}

\title{
1 Introduction
}

Primal-dual interior-point algorithms, originally introduced by Megiddo [10] in 1986 for linear programming, have a close connection to Newton's method. Exploiting this connection, Zhang, Tapia and Dennis [16] established sufficient conditions for primal-dual algorithms to achieve superlinear convergence for linear programming (LP). Zhang, Tapia and Potra [17] extended these results to quadratic programming (QP) and linear complementarity problems (LCP). Recently, Zhang and Tapia [18] constructed a primal-dual algorithm that exhibits both polynomial complexity and $Q$-superlinear convergence $(Q$ quadratic in the nondegenerate case). More recently, Kojima, Kurita and Mizuno [2] proposed rules for choosing parameters in primal-dual algorithms, and showed that for LCP one set of rules leads to both global convergence (but not necessarily polynomial) and superlinear convergence.

In this paper, we extend the Zhang-Tapia algorithm [18] from LP to QP and LCP, and prove the same theoretical properties, i.e., polynomial complexity and superlinear convergence, for the extended algorithm.

We take a unified approach towards linear programming quadratic programming and linear complementarity problems. The basis of this unified approach is the following nonlinear system with nonnegativity restrictions on the variables

$$
F(x, y)=\left(\begin{array}{c}
M x+N y-h \\
X Y e
\end{array}\right)=0, \quad(x, y) \geq 0,
$$


where $x, y, h, e \in \mathbf{R}^{n}, M, N \in \mathbf{R}^{n \times n}, X=\operatorname{diag}(x), Y=\operatorname{diag}(y)$ and $e$ has all components equal to one.

Problem (1.1) is sufficiently general to include linear complementarity problems, quadratic programming problems and linear programming problems. To begin with, observe that if $N=-I$, then this problem is the standard linear complementarity problem. Consider the following equality constrained quadratic programming problem

$$
\begin{array}{ll}
\operatorname{minimize} & c^{T} x+\frac{1}{2} x^{T} Q x \\
\text { subject to } & A x=b, \\
& x \geq 0
\end{array}
$$

where $c, x \in \mathbf{R}^{n}, b \in \mathbf{R}^{m}, A \in \mathbf{R}^{m \times n}(m<n)$ and has full row rank, and $Q \in \mathbf{R}^{n \times n}$. If $Q$ is symmetric and positive semi-definite on the null space of $A$; then $\mathrm{QP}(1.2)$ is a convex program and the first order optimality conditions are both necessary and sufficient for optimality. The first-order conditions for (1.2) can be transformed into the form of (1.1), as was shown in [17], with

$$
M=\left[\begin{array}{c}
A \\
-B Q
\end{array}\right], N=\left[\begin{array}{c}
0 \\
B
\end{array}\right] \text { and } h=\left(\begin{array}{c}
b \\
B c
\end{array}\right)
$$

where $B \in \mathbf{R}^{(n-m) \times n}$ is any matrix such that the columns of $B^{T}$ form a basis for the null space of $A$. When $Q=0$, the quadratic programming problem (1.2) reduces to the standard form linear programming problem.

A direct calculation shows that

$$
F^{\prime}(x, y)=\left[\begin{array}{ll}
M & N \\
Y & X
\end{array}\right]
$$

The feasibility set of problem (1.1) is:

$$
\mathcal{F}=\left\{(x, y): x, y \in \mathbf{R}^{n}, M x+N y=h,(x, y) \geq 0\right\}
$$

Moreover, a feasible pair $(x, y) \in \mathcal{F}$ is said to be strictly feasible if it is positive. 
Throughout this paper, whenever we consider LCP, we assume that the matrix $M$ is positive semi-definite; and whenever we consider $\mathrm{QP}$, we assume that the matrix $Q$ is symmetric and positive semi-definite on the null space of $A$. Also, we will assume that a strictly feasible point exists.

Subscripts will be used to distinguish values of quantities at a particular iteration and superscripts will indicate components of vectors. We also use the notation:

$$
\min (v) \equiv \min \left(v^{i}\right) \equiv \min _{1 \leq i \leq n} v^{i} \text { and } \max (v) \equiv \max \left(v^{i}\right) \equiv \max _{1 \leq i \leq n} v^{i}
$$

for a vector $v \in \mathbf{R}^{n}$. The symbol $\|\cdot\|$ denotes the $\ell_{2}$ norm unless otherwise stated.

The paper is organized as follows. In Section 2, we describe the basic interior-point algorithm for our general model (1.1). In Sections 3, we specify our choice of the step-length and the centering parameter. Polynomiality and superlinear convergence are established in Sections 4 and 5, respectively. Concluding remarks are given in Section 6.

\section{Algorithm}

The basic algorithm we will study is the following.

\section{Algorithm 1}

Given a strictly feasible pair $\left(x_{0}, y_{0}\right)$. For $k=0,1,2, \ldots$, do

Step 1 Compute the Newton step

$$
\left(\begin{array}{c}
\Delta x_{k}^{N} \\
\Delta y_{k}^{N}
\end{array}\right)=-\left[F^{\prime}\left(x_{k}, y_{k}\right)\right]^{-1} F\left(x_{k}, y_{k}\right)
$$

and the centering step

$$
\left(\begin{array}{c}
\Delta x_{k}^{C} \\
\Delta y_{k}^{C}
\end{array}\right)=\frac{1}{n} x_{k}^{T} y_{k}\left[F^{\prime}\left(x_{k}, y_{k}\right)\right]^{-1}\left(\begin{array}{l}
0 \\
e
\end{array}\right) .
$$


Step 2 Choose $\sigma_{k} \in(0,1)$ and form the combined step

$$
\left(\begin{array}{c}
\Delta x_{k} \\
\Delta y_{k}
\end{array}\right)=\left(\begin{array}{c}
\Delta x_{k}^{N} \\
\Delta y_{k}^{N}
\end{array}\right)+\sigma_{k}\left(\begin{array}{c}
\Delta x_{k}^{C} \\
\Delta y_{k}^{C}
\end{array}\right) .
$$

Step 3 Choose $\alpha_{k}=\tau_{k} \hat{\alpha}_{k}$, where $\tau_{k} \in(0,1)$ and

$$
\hat{\alpha}_{k}=\frac{-1}{\min \left(X_{k}^{-1} \Delta x_{k}, Y_{k}^{-1} \Delta y_{k}\right)} .
$$

Step 4 Compute the new iterate

$$
\left(\begin{array}{c}
x_{k+1} \\
y_{k+1}
\end{array}\right)=\left(\begin{array}{c}
x_{k} \\
y_{k}
\end{array}\right)+\alpha_{k}\left(\begin{array}{c}
\Delta x_{k} \\
\Delta y_{k}
\end{array}\right) .
$$

Notice that since $\left(x_{0}, y_{0}\right)$ is strictly feasible, all the subsequent iterates $\left\{\left(x_{k}, y_{k}\right)\right\}$ will also be strictly feasible. Also for QP and LCP, the matrix $F^{\prime}(x, y)$ is nonsingular for all $(x, y)>0$; hence Algorithm 1 is well-defined.

For $(x, y) \in \mathcal{F}$, we use the $\ell_{1}$-norm of the residual of $(1.1)$, i.e.,

$$
\|F(x, y)\|_{1}=x^{T} y
$$

as our measure of convergence. This measure can be shown to be the duality gap in linear and quadratic programming.

Observe that all three pairs $\left(\Delta x_{k}, \Delta y_{k}\right),\left(\Delta x_{k}^{N}, \Delta y_{k}^{N}\right)$ and $\left(\Delta x_{k}^{C}, \Delta y_{k}^{C}\right)$ satisfy the equation $M u+N v=0$. We also have the following useful relationships:

$$
Y_{k} \Delta x_{k}+X_{k} \Delta y_{k}=-X_{k} Y_{k} e+\sigma_{k} \frac{1}{n} x_{k}^{T} y_{k} e
$$

and

$$
x_{k+1}^{T} y_{k+1}=x_{k}^{T} y_{k}\left(1-\left(1-\sigma_{k}\right) \alpha_{k}\right)+\Delta x_{k}^{T} \Delta y_{k} \alpha_{k}^{2} .
$$

Algorithm 1 covers, or is closely related to, a wide range of existing interior-point algorithms for linear programming, quadratic programming and linear complementarity 
problems. In particular, it covers most of the existing primal-dual interior-point algorithms for linear programming as well as quadratic programming, including Kojima, Mizuno and Yoshise [8], Todd and Ye [13], Monteiro and Adler [11, 12], Lustig [9], to name a few. Algorithms for linear complementarity problems that are covered by Algorithm 1 include Kojima, Mizuno and Yoshise [6, 7], Kojima, Megiddo and Noma [3], Kojima, Megiddo and Ye [4], Kojima, Mizuno and Noma [5]. For more references, see two recent review papers by Ye $[14,15]$. Although these algorithms have been motivated and presented in various ways including path-following (homotopy or continuation), potential reduction or affine scaling algorithms, most of them fit into the framework of Algorithm 1.

\section{Choices of Parameters}

There are two control parameters in Algorithm 1. One is the so-called centering parameter $\sigma_{k}$ and the other is the step-length parameter $\alpha_{k}$. In [17], Zhang, Tapia and Potra established sufficient conditions for choosing the two parameters for Algorithm 1 to achieve superlinear convergence. Our concern in this paper is both global and local behavior of the algorithm appled to QP and LCP. For LP, this issue has been studied by Zhang and Tapia [18]. They gave rules for choosing the centering and the step-length parameters so that both polynomial complexity and superlinear convergence are obtained. In this section, we will modify their rules for choosing the step-length parameter for LP. The modified rules will fit not only LP but also QP and LCP.

We adopt the following notation:

$$
\begin{array}{ll}
x_{k}(\alpha)=x_{k}+\alpha \Delta x_{k}, & y_{k}(\alpha)=y_{k}+\alpha \Delta y_{k}, \\
f_{k}(\alpha)=X_{k}(\alpha) Y_{k}(\alpha) e, & f_{k}^{\text {ave }}(\alpha)=\frac{1}{n} x_{k}(\alpha)^{T} y_{k}(\alpha), \\
f_{k}^{\min }(\alpha)=\min \left(f_{k}(\alpha)\right), & f_{k}^{\max }(\alpha)=\max \left(f_{k}(\alpha)\right) .
\end{array}
$$

Whenever $\alpha=0$, we will drop the argument from the above functions. For example, $x_{k} \equiv$ 
$x_{k}(0), f_{k}^{\text {ave }} \equiv f_{k}^{\text {ave }}(0)$ and so on. Clearly, we also have $x_{k+1}=x_{k}\left(\alpha_{k}\right), f_{k+1}^{\text {ave }}=f_{k}^{\text {ave }}\left(\alpha_{k}\right)$ and so on.

It is important not to allow any $x_{k}^{i} y_{k}^{i}$ to become too small compared with the average value $\frac{1}{n} x_{k}^{T} y_{k}$ (see [17], for example). Hence we require that $\alpha=\alpha_{k}$ satisfies

$$
\frac{f_{k}^{\min }(\alpha)}{f_{k}^{\text {ave }}(\alpha)} \geq \gamma_{k}, \quad \alpha>0
$$

where

$$
\gamma_{k} \in\left[\gamma, f_{k}^{\min } / f_{k}^{\text {ave }}\right] \text { and } 0<\gamma \leq f_{0}^{\min } / f_{0}^{\text {ave }} \leq 1 \text {. }
$$

Note that in the case $f_{0}^{\min } / f_{0}^{\text {ave }}>\gamma$, we allow $\gamma_{k}$ to decrease monotonically as long as $\gamma_{k}>\gamma$.

It is easy to verify that

$$
f_{k}^{i}(\alpha)=f_{k}^{i}-\left(f_{k}^{i}-\sigma_{k} f_{k}^{\text {ave }}\right) \alpha+\Delta x_{k}^{i} \Delta y_{k}^{i} \alpha^{2} .
$$

Hence, $f_{k}^{i}(\alpha)$ is a quadratic for every $i$. As a result, $f_{k}^{\min }(\alpha)$ and $f_{k}^{\max }(\alpha)$ are piecewise quadratics. Moreover, $f_{k}^{\text {ave }}(\alpha)$ is also a quadratic and

$$
f_{k}^{\text {ave }}(\alpha)=f_{k}^{\text {ave }}\left[1-\left(1-\sigma_{k}\right) \alpha\right]+\frac{\Delta x_{k}^{T} \Delta y_{k}}{n} \alpha^{2} .
$$

For notational convenience, let us introduce the piecewise quadratic function

$$
h_{k}(\alpha)=f_{k}^{\min }(\alpha)-\gamma_{k} f_{k}^{\text {ave }}(\alpha) .
$$

It follows that condition (3.2) is equivalent to

$$
h_{k}(\alpha) \geq 0, \quad \alpha>0 .
$$

In determining $\alpha_{k}$ we will use the following quantity:

$$
\alpha_{k}^{\gamma}=\min \left\{\alpha>0: h_{k}(\alpha)=0\right\}
$$

The following lemma is given in Zhang and Tapia [18]. 
Lemma 3.1 The quantity $\alpha_{k}^{\gamma}$ is well defined and $\alpha_{k}^{\gamma} \in\left(0, \hat{\alpha}_{k}\right)$. Moreover, condition (3.2) is satisfied for all $\alpha \in\left(0, \alpha_{k}^{\gamma}\right]$.

An equivalent expression for $\alpha_{k}^{\gamma}$ is

$$
\alpha_{k}^{\gamma}=\min \left\{\alpha>0: f_{k}^{i}(\alpha)-\gamma_{k} f_{k}^{\text {ave }}(\alpha)=0, i=1,2, \ldots, n\right\} .
$$

The computation of $\alpha_{k}^{\gamma}$ involves calculating the roots of at most $n$ quadratics and therefore requires $O(n)$ operations.

In addition to a lower bound for $\left\{f_{k}^{i}\left(\alpha_{k}\right) / f_{k}^{\text {ave }}\left(\alpha_{k}\right)\right\}$ (i.e., condition (3.2)), we also impose an upper bound on these quantities; namely, we require $\alpha_{k}$ to satisfy

$$
\frac{f_{k}^{\max }(\alpha)}{f_{k}^{\text {ave }}(\alpha)} \leq \Gamma_{k}, \quad \alpha>0
$$

where

$$
\Gamma_{k} \in\left[f_{k}^{\max } / f_{k}^{\text {ave }}, \Gamma\right] \text { and } 1 \leq f_{0}^{\max } / f_{0}^{\text {ave }} \leq \Gamma \leq n .
$$

Since $f_{k}^{i}(\alpha) / f_{k}^{\text {ave }}(\alpha)<n$ for all $i$, condition (3.9) will be redundant if $\Gamma_{k}=n$. Our reason for introducing condition (3.9) is to improve our complexity bound.

Similarly, we introduce the piecewise quadratic function

$$
H_{k}(\alpha)=f_{k}^{\max }(\alpha)-\Gamma_{k} f_{k}^{\text {ave }}(\alpha) .
$$

It is easy to see that condition (3.9) is equivalent to

$$
H_{k}(\alpha) \leq 0, \quad \alpha>0 \text {. }
$$

We define

$$
\alpha_{k}^{\Gamma}=\left\{\begin{array}{l}
+\infty, \text { if }\left\{\alpha>0: H_{k}(\alpha)=0\right\}=\emptyset \\
\min \left\{\alpha>0: H_{k}(\alpha)=0\right\}, \text { otherwise. }
\end{array}\right.
$$

Analogous to Lemma 3.1 for condition (3.2), we have the following lemma for condition (3.9).

Lemma 3.2 The quantity $\alpha_{k}^{\Gamma}$ is well-defined and $\alpha_{k}^{\Gamma} \in\left(0, \hat{\alpha}_{k}\right)$. Moreover, condition (3.9) is satisfied by all $\alpha \in\left(0, \alpha_{k}^{\Gamma}\right)$ and also by $\alpha=\alpha_{k}^{\Gamma}$ when the latter is finite. 
Proof: The proof is similar to that for Lemma 3.1, so we omit it.

Similarly, $\alpha_{k}^{\Gamma}$ can be found by calculating the roots of at most $n$ quadratics and requires $O(n)$ operations.

The duality gap

$$
x_{k}(\alpha)^{T} y_{k}(\alpha)=x_{k}^{T} y_{k}-x_{k}^{T} y_{k}\left(1-\sigma_{k}\right) \alpha+\Delta x_{k}^{T} \Delta y_{k} \alpha^{2}
$$

is a quadratic function of $\alpha$ with the second derivative $\Delta x_{k}^{T} \Delta y_{k}$. If $\Delta x_{k}^{T} \Delta y_{k}>0$, it will reach its minimum at

$$
\alpha_{k}^{\nu}=\frac{\left(1-\sigma_{k}\right) x_{k}^{T} y_{k}}{2 \Delta x_{k}^{T} \Delta y_{k}}
$$

If $\Delta x_{k}^{T} \Delta y_{k}=0$, the duality gap is a decreasing function of $\alpha$; so it is always desirable to take the largest step-length possible. For LCP and QP, one always has $\Delta x_{k}^{T} \Delta y_{k} \geq 0$ (see Lemma 3.3 below). Therefore our choice of the step-length $\alpha_{k}$ is

$$
\alpha_{k}=\left\{\begin{array}{cl}
\min \left(1, \alpha_{k}^{\gamma}, \alpha_{k}^{\Gamma}, \alpha_{k}^{\nu}\right), & \text { if } \Delta x_{k}^{T} \Delta y_{k}>0 \\
\min \left(1, \alpha_{k}^{\gamma}, \alpha_{k}^{\Gamma}\right), & \text { otherwise }
\end{array}\right.
$$

where $\alpha_{k}^{\gamma}$ is defined by (3.8), $\alpha_{k}^{\Gamma}$ by (3.13) and $\alpha_{k}^{\nu}$ by (3.15). This choice of step-length will guarantee $(3.2),(3.9)$ and $\alpha_{k} \in\left(0, \hat{\alpha}_{k}\right)$.

For the sake of simplicity, we will enforce the conditions

$$
\gamma_{k} \leq 1 / 2 \text { and } \Gamma_{k} \geq 2
$$

The specific values in (3.17) do not constitute a loss of generality because they will only affect expressions for some constants in our analysis. These values of $\gamma_{k}$ and $\Gamma_{k}$ will lead to much simplified expressions for those constants.

\section{Procedure 1}

Given

$$
0<\gamma \leq \min \left(1 / 2, f_{0}^{\min } / f_{0}^{\text {ave }}\right), \max \left(2, f_{0}^{\max } / f_{0}^{\text {ave }}\right) \leq \Gamma<n
$$

Step 1 Choose $\gamma_{k} \in\left[\gamma, \min \left(1 / 2, f_{k}^{\min } / f_{k}^{\text {ave }}\right)\right]$ and $\Gamma_{k} \in\left[\max \left(2, f_{k}^{\max } / f_{k}^{\text {ave }}\right), \Gamma\right]$. 
Step 2 Compute $\alpha_{k}^{\gamma}$ by (3.8), $\alpha_{k}^{\Gamma}$ by (3.13), and if necessary, $\alpha_{k}^{\nu}$ by (3.15).

Step 3 Compute $\alpha_{k}$ by (3.16).

When $\Delta x_{k}^{T} \Delta y_{k}=0$, the above procedure basically reduces to Procedure 1 in [18], which was designed for LP where $\Delta x_{k}^{T} \Delta y_{k} \equiv 0$.

Now we shift our attention to the choice of the centering parameter. We will use exactly the same strategy to choose $\sigma_{k}$ as that used in [18]. However, for the sake of completeness we still describe the choice in detail. Let

$$
\begin{aligned}
& p_{k}=X_{k}^{-1} \Delta x_{k}, \quad q_{k}=Y_{k}^{-1} \Delta y_{k}, \\
& p_{k}^{N}=X_{k}^{-1} \Delta x_{k}^{N}, \quad q_{k}^{N}=Y_{k}^{-1} \Delta y_{k}^{N}, \\
& p_{k}^{C}=X_{k}^{-1} \Delta x_{k}^{C}, \quad q_{k}^{C}=Y_{k}^{-1} \Delta y_{k}^{C},
\end{aligned}
$$

and

$$
\left.\omega_{k}=\max _{1 \leq i \leq n}\left|\left(p_{k}^{N}\right)^{i}\left(q_{k}^{N}\right)^{i}\right|,\left|\left(p_{k}^{N}\right)^{i}\left(q_{k}^{C}\right)^{i}\right|,\left|\left(p_{k}^{C}\right)^{i}\left(q_{k}^{N}\right)^{i}\right|,\left|\left(p_{k}^{C}\right)^{i}\left(q_{k}^{C}\right)^{i}\right|\right) .
$$

Lemma 3.3 For QP and LCP,

(i) $\Delta x_{k}^{T} \Delta y_{k} \geq 0,\left(\Delta x_{k}^{N}\right)^{T}\left(\Delta y_{k}^{N}\right) \geq 0$ and $\left(\Delta x_{k}^{N}\right)^{T}\left(\Delta y_{k}^{N}\right) \geq 0$;

(ii) if $f_{k}^{\min } / f_{k}^{\text {ave }} \geq \gamma$, then $\omega_{k} \leq n / \gamma^{2}$.

Proof: (i) Since all three pairs $\left(\Delta x_{k}, \Delta y_{k}\right),\left(\Delta x_{k}^{N}, \Delta y_{k}^{N}\right)$ and $\left(\Delta x_{k}^{C}, \Delta y_{k}^{C}\right)$ satisfy the equation $M u+N v=0$. It suffices to prove that $u^{T} v \geq 0$ for all $u, v \in \mathbf{R}^{n}$ satisfying $M u+N v=0$.

In the case of $\operatorname{LCP}(N=-I), M u+N v=0$ is equivalent to $v=M u$. Hence $u^{T} v=u^{T} M u \geq 0$ because $M$ is positive semi-definite.

In the case of $\mathrm{QP}, M u+N v=0$ is equivalent to $A u=0$ and $B Q u=B v$ (see (1.3)). Using the representations $u=B^{T} u_{2}$ and $v=A^{T} v_{1}+B^{T} v_{2}$, where $v_{1} \in \mathbf{R}^{m}$ and $u_{2}, v_{2} \in \mathbf{R}^{n-m}$, and noticing that $A^{T} \perp B^{T}$, we have $u^{T} v=u_{2}^{T} B B^{T} v_{2}$. Moreover, 
$B Q u=B v$ is equivalent to $B Q B^{T} u_{2}=B B^{T} v_{2}$. Hence, if $Q$ is positive semi-definite on the null space of $A$, then

$$
u^{T} v=u_{2}^{T} B B^{T} v_{2}=u_{2}^{T}\left(B Q B^{T}\right) u_{2} \geq 0 .
$$

(ii) Multiply both sides of $Y_{k} \Delta x_{k}^{N}+X_{k} \Delta y_{k}^{N}=-X_{k} Y_{k} e$ by $\left(X_{k} Y_{k}\right)^{-\frac{1}{2}}$ and consider the square of the $\ell_{2}$-norm of both sides. Using (i) of Lemma 3.3, we have

$$
\left\|\left(X_{k} Y_{k}\right)^{\frac{1}{2}} p_{k}^{N}\right\|^{2}+\left\|\left(X_{k} Y_{k}\right)^{\frac{1}{2}} q_{k}^{N}\right\|^{2} \leq x_{k}^{T} y_{k}
$$

or equivalently after dividing both sides by $\frac{1}{n} x_{k}^{T} y_{k}$,

$$
\left\|T_{k}^{-\frac{1}{2}} p_{k}^{N}\right\|^{2}+\left\|T_{k}^{-\frac{1}{2}} q_{k}^{N}\right\|^{2} \leq n
$$

where $T_{k}=\frac{1}{n} x_{k}^{T} y_{k}\left(X_{k} Y_{k}\right)^{-1}$ is a diagonal matrix. Since $f_{k}^{\min } / f_{k}^{\text {ave }} \geq \gamma$, it is easy to see that

$$
\left|\left(p_{k}^{N}\right)^{i}\right| \leq \sqrt{n / \gamma} \leq \sqrt{n} / \gamma \text { and }\left|\left(q_{k}^{N}\right)^{i}\right| \leq \sqrt{n / \gamma} \leq \sqrt{n} / \gamma .
$$

Similarly, we can prove

$$
\left|\left(p_{k}^{C}\right)^{i}\right| \leq \sqrt{n} / \gamma \text { and }\left|\left(q_{k}^{C}\right)^{i}\right| \leq \sqrt{n} / \gamma
$$

The result in (ii) of Lemma 3.3 follows directly from the definition of $\omega_{k}$ and the above estimates.

We now state our procedure for choosing the centering parameter $\sigma_{k}$.

\section{Procedure 2}

Given

$$
\sigma \in(0,1), \quad \rho^{l}=\frac{\gamma^{2} \sigma}{2 n}, \quad \rho^{u} \geq \frac{\gamma^{2} \sigma}{n} .
$$

Step 1 Compute $\omega_{k}$ from (3.20).

Step 2 Compute $\rho_{k}^{u}=\min \left(\rho^{u}, \sigma / \omega_{k}\right)$.

Step 3 Choose $\rho_{k} \in\left[\left(\rho^{l}+\rho_{k}^{u}\right) / 2, \rho_{k}^{u}\right]$. 
Step 4 Let $\sigma_{k}=\rho_{k} \omega_{k}$.

It is easy to verify, using (ii) of Lemma 3.3 , that $\rho_{k}^{u}>\rho^{l}$; thus $\sigma_{k}$ is well-defined. Moreover, we have $\sigma_{k} \leq \rho_{k}^{u} \omega_{k} \leq \sigma<1$.

Overall, we have the following algorithm.

Algorithm 2 Given a strictly feasible pair $\left(x_{0}, y_{0}\right)$. Choose (recall (3.18))

$$
0<\gamma \leq \min \left(1 / 2, f_{0}^{\min } / f_{0}^{\text {ave }}\right), \max \left(2, f_{0}^{\max } / f_{0}^{\text {ave }}\right) \leq \Gamma<n,
$$

and $\sigma \in(0,1)$. Set $\rho^{l}=\gamma^{2} \sigma / 2 n$ and $\rho^{u} \geq \gamma^{2} \sigma / n$. For $k=0,1,2, \ldots$, do

Step 1 Compute $\left(\Delta x_{k}^{N}, \Delta y_{k}^{N}\right)$ and $\left(\Delta x_{k}^{C}, \Delta y_{k}^{C}\right)$ from Algorithm 1.

Step 2 Choose $\sigma_{k}$ by Procedure 2 and form $\left(\Delta x_{k}, \Delta y_{k}\right)$ from Algorithm 1.

Step 3 Choose $\alpha_{k}$ by Procedure 1.

Step 4 Form $\left(x_{k+1}, y_{k+1}\right)$ from Algorithm 1.

\section{Polynomiality}

In this section, we will establish the global behavior of Algorithm 2. We first define

$$
\begin{aligned}
\xi_{k} & =\frac{\Gamma_{k}}{n} \Delta x_{k}^{T} \Delta y_{k}-\max \left(\Delta x_{k}^{i} \Delta y_{k}^{i}\right) \\
\eta_{k} & =\frac{\gamma_{k}}{n} \Delta x_{k}^{T} \Delta y_{k}-\min \left(\Delta x_{k}^{i} \Delta y_{k}^{i}\right) .
\end{aligned}
$$

Lemma 4.1 Let $\alpha_{k}^{\gamma}$ be given by (3.8). If $\eta_{k} \leq 0$, then $\alpha_{k}^{\gamma} \geq 1$; otherwise,

$$
\alpha_{k}^{\gamma} \geq \frac{\left(1-\gamma_{k}\right) \sigma_{k} x_{k}^{T} y_{k}}{n \eta_{k}}=\frac{\left(1-\gamma_{k}\right) \sigma_{k} f_{k}^{\text {ave }}}{\eta_{k}}
$$

Proof: First we notice that for $\alpha \in[0,1]$,

$$
f_{k}^{i}(\alpha)=f_{k}^{i}-\left(f_{k}^{i}-\sigma_{k} f_{k}^{\text {ave }}\right) \alpha+\Delta x_{k}^{i} \Delta y_{k}^{i} \alpha^{2} \geq f_{k}^{\min }-\left(f_{k}^{\min }-\sigma_{k} f_{k}^{\text {ave }}\right) \alpha+\min \left(\Delta x_{k}^{i} \Delta y_{k}^{i}\right) \alpha^{2} .
$$


Therefore,

$$
\begin{aligned}
f_{k}^{i}(\alpha)-\gamma_{k} f_{k}^{\text {ave }}(\alpha) & \geq f_{k}^{\text {min }}-\left(f_{k}^{\text {min }}-\sigma_{k} f_{k}^{\text {ave }}\right) \alpha+\min \left(\Delta x_{k}^{i} \Delta y_{k}^{i}\right) \alpha^{2}-\gamma_{k} f_{k}^{\text {ave }}(\alpha) \\
& =\left(f_{k}^{\text {min }}-\gamma_{k} f_{k}^{\text {ave }}\right)(1-\alpha)+\left(1-\gamma_{k}\right) \sigma_{k} f_{k}^{\text {ave }} \alpha-\eta_{k} \alpha^{2} \\
& \geq\left(1-\gamma_{k}\right) \sigma_{k} f_{k}^{\text {ave }} \alpha-\eta_{k} \alpha^{2}
\end{aligned}
$$

If $\eta_{k} \leq 0$, then it follows that $h_{k}(\alpha)$ has no root in $(0,1)$; hence $\alpha_{k}^{\gamma} \geq 1$. Otherwise, the quadratic in the right-hand side of the last inequality has a unique positive root

$$
\alpha=\frac{\left(1-\gamma_{k}\right) \sigma_{k} x_{k}^{T} y_{k}}{n \eta_{k}}=\frac{\left(1-\gamma_{k}\right) \sigma_{k} f_{k}^{\text {ave }}}{\eta_{k}}
$$

Therefore, if $\alpha_{k}^{\gamma}<1$, from (4.3) $\alpha_{k}^{\gamma}$ must be greater than or equal to the above positive number. This completes the proof.

Lemma 4.2 Let $\alpha_{k}^{\Gamma}$ be given by (3.13). If $\xi_{k} \geq 0$, then $\alpha_{k}^{\Gamma} \geq 1$; otherwise,

$$
\alpha_{k}^{\Gamma} \geq \frac{\left(1-\Gamma_{k}\right) \sigma_{k} x_{k}^{T} y_{k}}{n \xi_{k}}=\frac{\left(1-\Gamma_{k}\right) \sigma_{k} f_{k}^{\text {ave }}}{\xi_{k}}
$$

Proof: The proof is similar to that of Lemma 4.1. So we omit it.

Lemma 4.3 Let $\xi_{k}$ and $\eta_{k}$ be given by (4.1).

(i) $\xi_{k} \geq-4 \omega_{k} f_{k}^{\max }$

(ii) $\eta_{k} \leq 6 \omega_{k} f_{k}^{\max }$.

Proof: (i) Note that

$$
\begin{aligned}
\left|\Delta x_{k}^{i} \Delta y_{k}^{i}\right| & =\left|\left(x_{k}^{i} y_{k}^{i}\right)\left(p_{k}^{i} q_{k}^{i}\right)\right| \\
& \leq \max \left(X_{k} Y_{k} e\right)\left\|\operatorname{diag}\left(p_{k}\right) q_{k}\right\|_{\infty} \\
& =f_{k}^{\max }\left\|\operatorname{diag}\left(p_{k}^{N}+\sigma_{k} p_{k}^{C}\right)\left(q_{k}^{N}+\sigma_{k} p_{k}^{C}\right)\right\|_{\infty} \\
& \leq f_{k}^{\max } \omega_{k}\left(1+\sigma_{k}\right)^{2} \\
& \leq 4 f_{k}^{\max } \omega_{k} .
\end{aligned}
$$


Since $\Delta x_{k}^{T} \Delta y_{k} \geq 0$, we have (see (4.1))

$$
\xi_{k} \geq-\max \left(\Delta x_{k}^{i} \Delta y_{k}^{i}\right) \geq-4 f_{k}^{\max } \omega_{k}
$$

(ii) Since $\left|\Delta x_{k}^{i} \Delta y_{k}^{i}\right| \leq 4 f_{k}^{\max } \omega_{k}, \Delta x_{k}^{T} \Delta y_{k} \leq 4 n f_{k}^{\max } \omega_{k}$. Therefore,

$$
\begin{aligned}
\eta_{k} & =\frac{\gamma_{k}}{n} \Delta x_{k}^{T} \Delta y_{k}-\min \left(\Delta x_{k}^{i} \Delta y_{k}^{i}\right) \\
& \leq 4 \gamma_{k} f_{k}^{\max } \omega_{k}+4 f_{k}^{\max } \omega_{k} \\
& \leq 6 f_{k}^{\max } \omega_{k}
\end{aligned}
$$

because $\gamma_{k} \leq 1 / 2$. This completes the proof.

Lemma 4.4 Let $\left(x_{k}, y_{k}\right),\left(\Delta x_{k}, \Delta y_{k}\right)$ and $\left(\alpha_{k}^{\gamma}, \alpha_{k}^{\Gamma}\right)$ be generated by Algorithm 2. Then there exists a constant $\beta>0$, such that

$$
\min \left(1, \alpha_{k}^{\gamma}, \alpha_{k}^{\Gamma}\right) \geq \frac{\beta}{n}
$$

Moreover,

$$
\frac{\Delta x_{k}^{T} \Delta y_{k}}{x_{k}^{T} y_{k}} \leq \frac{1}{4 \gamma}
$$

Proof: From Lemmas 4.1 and 4.2, it suffices to consider the situation where $\xi_{k}<0$ and $\eta_{k}>0$. In this case,

$$
\begin{aligned}
& \min \left(1, \alpha_{k}^{\gamma}, \alpha_{k}^{\Gamma}\right) \geq \min \left(1, \frac{\left(1-\gamma_{k}\right) \sigma_{k} f_{k}^{\text {ave }}}{\eta_{k}}, \frac{\left(1-\Gamma_{k}\right) \sigma_{k} f_{k}^{\text {ave }}}{\xi_{k}}\right) \text { [Lemmas 4.1-4.2] } \\
& \geq \min \left(1, \frac{0.5 \sigma_{k} f_{k}^{\text {ave }}}{6 \omega_{k} f_{k}^{\max }}, \frac{\sigma_{k} f_{k}^{\text {ave }}}{4 \omega_{k} f_{k}^{\max }}\right) \quad[(3.17) \text { and Lemma } 4.3] \\
& \geq \min \left(1, \frac{\rho_{k}}{12 \Gamma}\right) \quad\left[f_{k}^{\max } / f_{k}^{\text {ave }} \leq \Gamma \text { and } \sigma_{k}=\rho_{k} \omega_{k}\right] \\
& \geq \min \left(1, \frac{\gamma^{2} \sigma}{24 \Gamma_{n}}\right) \quad\left[\rho_{k} \geq \rho^{l}=\gamma^{2} \sigma / 2 n\right] \\
& \geq\left(\frac{\gamma^{2} \sigma}{24 \Gamma}\right) \frac{1}{n} \quad[\gamma<1, \Gamma>1 \text { and } \sigma<1] \text {. }
\end{aligned}
$$

Hence,

$$
\beta=\frac{\gamma^{2} \sigma}{24 \Gamma}
$$

will satisfies (4.5). 
Now define $D_{k}=X_{k} Y_{k}^{-1}$ and let

$$
r_{k}=\left[\sigma_{k} \frac{1}{n} x_{k}^{T} y_{k}\left(X_{k} Y_{k}\right)^{-1 / 2}-\left(X_{k} Y_{k}\right)^{1 / 2}\right] e
$$

It follows that

$$
r_{k}^{T} r_{k}=x_{k}^{T} y_{k}\left(1-2 \sigma_{k}+\sigma_{k}^{2} \frac{1}{n} x_{k}^{T} y_{k} \frac{e^{T}\left(X_{k} Y_{k}\right)^{-1} e}{n}\right) \leq x_{k}^{T} y_{k}\left(1-2 \sigma_{k}+\sigma_{k}^{2} / \gamma\right)
$$

since $f_{k}^{\text {ave }} / f_{k}^{i} \leq 1 / \gamma$.

Multiplying both sides of $(2.1)$ by $\left(X_{k} Y_{k}\right)^{-1 / 2}$ gives

$$
r_{k}=D_{k}^{-1 / 2} \Delta x_{k}+D_{k}^{1 / 2} \Delta y_{k}
$$

Therefore,

$$
\begin{aligned}
r_{k}^{T} r_{k} & =\Delta x_{k}^{T} D_{k}^{-1} \Delta x_{k}+\Delta y_{k}^{T} D_{k} \Delta y_{k}+2 \Delta x_{k}^{T} \Delta y_{k} \\
& =\left\|D_{k}^{-1 / 2} \Delta x_{k}-D_{k}^{1 / 2} \Delta y_{k}\right\|^{2}+4 \Delta x_{k}^{T} \Delta y_{k} \\
& \geq 4 \Delta x_{k}^{T} \Delta y_{k} .
\end{aligned}
$$

Combining (4.8) and (4.9) and noting $\sigma_{k}<1$ leads to

$$
\begin{aligned}
\frac{\Delta x_{k}^{T} \Delta y_{k}}{x_{k}^{T} y_{k}} & \leq \frac{1}{4}\left(1-2 \sigma_{k}+\sigma_{k}^{2} / \gamma\right) \\
& =\frac{1}{4}\left(\left(1-\sigma_{k}\right)^{2}+\sigma_{k}^{2}\left(\frac{1}{\gamma}-1\right)\right) \\
& \leq \frac{1}{4 \gamma}
\end{aligned}
$$

This completes the proof.

\section{Theorem 4.1}

Let $\left\{\left(x_{k}, y_{k}\right)\right\}$ be generated by Algorithm 2. Then

$$
\frac{x_{k+1}^{T} y_{k+1}}{x_{k}^{T} y_{k}} \leq\left(1-\delta_{k}\right)<1
$$

where $\delta_{k}=\Omega\left(\frac{1}{n}\right)$

Proof: From (2.2),

$$
x_{k+1}^{T} y_{k+1}=x_{k}^{T} y_{k}\left(1-\left(1-\sigma_{k}\right) \alpha_{k}+\frac{\Delta x_{k}^{T} \Delta y_{k}}{x_{k}^{T} y_{k}} \alpha_{k}^{2}\right)
$$


We must estimate the quantity

$$
\delta_{k}=\left(1-\sigma_{k}-\frac{\Delta x_{k}^{T} \Delta y_{k}}{x_{k}^{T} y_{k}} \alpha_{k}\right) \alpha_{k}
$$

Since $\delta_{k}<1$ is guaranteed in Algorithm 2 by the choice of $\alpha_{k}$, it is sufficient to verify that $\delta_{k}=\Omega\left(\frac{1}{n}\right)$.

Consider the following two functions of $\alpha$

$$
\delta_{k}(\alpha)=\left(1-\sigma_{k}-\frac{\Delta x_{k}^{T} \Delta y_{k}}{x_{k}^{T} y_{k}} \alpha\right) \alpha
$$

and

$$
\bar{\delta}(\alpha)=\left(1-\sigma-\frac{1}{4 \gamma} \alpha\right) \alpha
$$

From (4.6) and $\sigma_{k} \leq \sigma$, for $\alpha \geq 0$

$$
\delta_{k}(\alpha) \geq \bar{\delta}(\alpha)
$$

From the choice of $\alpha_{k}$ in Procedure 1, it can be verified that

$$
\alpha_{k}=\arg \max \left\{\delta_{k}(\alpha): \alpha \in\left[0, \min \left(1, \alpha_{k}^{\gamma}, \alpha_{k}^{\Gamma}\right)\right]\right\}
$$

Therefore, for all $\alpha \in\left[0, \min \left(1, \alpha_{k}^{\gamma}, \alpha_{k}^{\Gamma}\right)\right]$,

$$
\delta_{k}=\delta_{k}\left(\alpha_{k}\right) \geq \bar{\delta}(\alpha)
$$

Let $\beta$ be defined by (4.7). It follows from (4.5) that (4.13) is satisfied by $\alpha=\beta / n$; i.e.,

$$
\delta_{k} \geq \bar{\delta}(\beta / n)=\left(1-\sigma-\frac{\beta}{4 \gamma n}\right) \frac{\beta}{n}=\Omega\left(\frac{1}{n}\right) .
$$

This completes the proof.

The following corollary follows immediately from Theorem 4.1. Its proof is straightforward (see [18]), for example). By a standard argument, it leads to polynomiality assuming integral data.

Corollary 4.1 Assume $x_{0}^{T} y_{0} \leq 2^{\nu L}$, where $L>0$ and $\nu>0$. Then in at most $O(n L)$ iterations, Algorithm 2 will produce $\left(x_{k}, y_{k}\right)$ satisfying $x_{k}^{T} y_{k} \leq 2^{-L}$. 


\section{Superlinear Convergence}

In this section, we will apply the following superlinear convergence result of Zhang, Tapia and Potra [17] to establish $Q$-superlinear convergence of Algorithm 2 for QP and LCP.

\section{Theorem 5.1 (Zhang, Tapia and Potra)}

Let $\left\{\left(x_{k}, y_{k}\right)\right\}$ be generated by Algorithm 1 and $\left(x_{*}, y_{*}\right)$ be a solution of problem (1.1). Assume

(i) strict complementarity holds at $\left(x_{*}, y_{*}\right)$,

(ii) $\left\{f_{k}^{\text {ave }} / f_{k}^{\min }\right\}$ is bounded above,

(iii) $\sigma_{k} \rightarrow 0$ and $\alpha_{k} \rightarrow 1$.

Then if $\left\{\left(x_{k}, y_{k}\right)\right\}$ converges to $\left(x_{*}, y_{*}\right)$, the sequence $\left\{X_{k} Y_{k} e\right\}$, component-wise, converges to zero Q-superlinearly. Moreover, under the above conditions, $\alpha_{k} \rightarrow 1$ if and only if $\tau_{k} \rightarrow 1$.

Since $\tau_{k}$ is directly under our control and $\alpha_{k}$ is not, the more meaningful result is stated in terms of $\tau_{k}$. However, Lemma 3.1 of Zhang, Tapia and Potra [17] demonstrates that under the conditions of the theorem, $\hat{\alpha}_{k}$ as defined in Step 3 of Algorithm 1 converges to 1 . Hence, $\alpha_{k} \rightarrow 1$ if and only if $\tau_{k} \rightarrow 1$ and we may consider either condition. In the sequel, we choose to verify $\alpha_{k} \rightarrow 1$.

We note that unlike in LP, a strictly complementary solution may not exist in $\mathrm{QP}$ or LCP. So the strict complementarity assumption for QP and LCP is a somewhat strong, but often made, assumption.

For the same technical reasons as encountered in Zhang and Tapia [18], we must restrict further the choice of $\rho_{k}$. Denote the length of the interval $\left[\rho^{l}, \rho_{k}^{u}\right]$ by $\pi_{k}$. It follows from (3.22), Step 2 of Procedure 2, and Lemma 3.3 that

$$
\rho_{k}^{u} \geq \frac{\gamma^{2} \sigma}{n}
$$


Thus,

$$
\pi_{k}=\rho_{k}^{u}-\rho^{l} \geq \frac{\gamma^{2} \sigma}{2 n}>0 .
$$

Let $\Sigma_{k}$ be the following set of $2 n$ points

$$
\Sigma_{k}=\left\{-\left(p_{k}^{N}\right)^{i} /\left(p_{k}^{C}\right)^{i},-\left(q_{k}^{N}\right)^{i} /\left(q_{k}^{C}\right)^{i}, i=1,2, \ldots, n\right\}
$$

and define the distance from $\sigma$ to the set $\Sigma_{k}$ as

$$
\operatorname{dist}\left(\sigma, \Sigma_{k}\right)=\min \left\{|\sigma-\varsigma|: \varsigma \in \Sigma_{k}\right\}
$$

We choose $\sigma_{k}$ according to Procedure 2 with the additional restriction that

$$
\operatorname{dist}\left(\sigma_{k}, \Sigma_{k}\right) \geq \pi_{k} \omega_{k} /(8 n+4)
$$

In other words, we require not only

$$
\sigma_{k} \in\left[0.5\left(\rho^{l}+\rho_{k}^{u}\right) \omega_{k}, \rho_{k}^{u} \omega_{k}\right]
$$

but also that $\sigma_{k}$ be bounded away from the set $\Sigma_{k}$ by at least the amount $\pi_{k} \omega_{k} /(8 n+4)$. Since $\left\{\pi_{k}\right\}$ is bounded away from zero, we see from (5.3) that $\left\{\operatorname{dist}\left(\sigma_{k}, \Sigma_{k}\right)\right\}$ is bounded away from zero when $\left\{\omega_{k}\right\}$ is bounded away from zero. It has been shown in Lemma 8.1 of Zhang and Tapia [18] that the set of $\sigma$ values satisfying (5.3) and (5.4) is nonempty.

Our superlinear convergence theory for Algorithm 2, applied to not only LP, but also to $\mathrm{QP}$ and LCP, is the following.

\section{Theorem 5.2}

Let $\left(x_{*}, y_{*}\right)$ be a solution of problem (1.1) and $\left\{\left(x_{k}, y_{k}\right)\right\}$ be generated by Algorithm 2 with the restriction (5.3) on the centering parameter $\sigma_{k}$. Assume

(i) strict complementarity holds at $\left(x_{*}, y_{*}\right)$,

(ii) $\rho^{u}$ is sufficiently large, e.g., $\rho^{u} \geq 24 \Gamma$. 
For $L P, Q P$ and $L C P$, if $\left\{\left(x_{k}, y_{k}\right)\right\}$ converges to $\left(x_{*}, y_{*}\right)$, then the sequence $\left\{X_{k} Y_{k} e\right\}$, component-wise, converges to zero Q-superlinearly.

Proof: Theorem 5.2 is a generalization of Theorem 8.1 in [18], i.e., the superlinear convergence of Algorithm 2 applied to LP. As for the proof of Theorem 8.1 in [18], we need to show $\sigma_{k} \rightarrow 0$ and $\alpha_{k} \rightarrow 1$.

The proof for $\sigma_{k} \rightarrow 0$ is identical to that in [18], because it only requires that $\left\{\alpha_{k}\right\}$ be bounded below and that $\left\{p_{k}^{N}\right\},\left\{q_{k}^{N}\right\},\left\{p_{k}^{C}\right\}$ and $\left\{q_{k}^{N}\right\}$ be bounded above, which are also true for QP and LCP (see Lemmas 3.3 and 4.4).

The proof for $\alpha_{k} \rightarrow 1$ is similar to that in [18]. First, the same argument used in [18] gives

$$
\alpha_{k} \leq \hat{\alpha}_{k} \rightarrow 1
$$

When $\Delta x_{k}^{T} \Delta y_{k}=0$, it follows from (3.16) and the proof of Lemma 4.4 that

$$
\alpha_{k}=\min \left(1, \alpha_{k}^{\gamma}, \alpha_{k}^{\Gamma}\right) \geq \min \left(1, \frac{\rho_{k}}{12 \Gamma}\right) .
$$

From the definition of $\rho_{k}$ and the fact $\omega_{k} \rightarrow 0$, for $k$ sufficiently large

$$
\rho_{k}=\frac{1}{2}\left(\rho^{l}+\rho_{k}^{u}\right) \geq \frac{1}{2} \rho_{k}^{u}=\frac{1}{2} \rho^{u} .
$$

Therefore, if $\rho^{u} \geq 24 \Gamma$, then from (5.6)

$$
\alpha_{k} \geq 1
$$

Now inequalities (5.5) and (5.7) together imply $\alpha_{k} \rightarrow 1$.

When $\Delta x_{k}^{T} \Delta y_{k}>0$, in order to prove (5.7) we also need to show that $\alpha_{k}^{\nu} \geq 1$ for $k$ sufficiently large (see (3.16)). It follows from $\Delta x_{k}^{T} \Delta y_{k} \leq 4 n \omega_{k} f_{k}^{\max }$ (see the proof of Lemma 4.3 (ii)), $f_{k}^{\max } / f_{k}^{\text {ave }} \leq \Gamma, \sigma_{k} \leq \sigma$ and $\omega_{k} \rightarrow 0$ that as $k \rightarrow \infty$

$$
\alpha_{k}^{\nu}=\frac{\left(1-\sigma_{k}\right) x_{k}^{T} y_{k}}{2 \Delta x_{k}^{T} \Delta y_{k}} \geq \frac{\left(1-\sigma_{k}\right) f_{k}^{\text {ave }}}{8 \omega_{k} f_{k}^{\max }} \geq \frac{1-\sigma}{8 \Gamma \omega_{k}} \rightarrow \infty .
$$

Hence, $\alpha_{k}=\min \left(1, \alpha_{k}^{\gamma}, \alpha_{k}^{\Gamma}, \alpha_{k}^{\nu}\right) \geq 1$ for $k$ sufficiently large. This completes the proof. 


\section{Concluding Remarks}

In this paper, we have constructed a primal-dual interior-point method for a general problem which includes linear programming, quadratic programming and linear complementarity problems, and have demonstrated its $O(n L)$-iteration polynomial complexity and its $Q$-superlinear convergence. We accomplished the above objectives by adopting the rules for choosing step-length in the Zhang-Tapia polynomial and superlinearly convergent algorithm for LP [18] to our general problem, and then extending their theory to our problem.

\section{Acknowledgment}

The authors thank Steve Wright for reading the first draft of this paper and spotting a number of misprints.

\section{References}

[1] G. B. Dantzig. Linear Programming and Extensions. Princeton University Press, Princeton, NJ, 1963.

[2] M. Kojima, Y. Kurita and S. Mizuno. Large-step interior point algorithms for linear complementarity problems. Research Report on Information Sciences B-243, Dept. of Information Sciences, Tokyo Institute of Technology, 1991.

[3] M. Kojima, N. Megiddo, and T. Noma. Homotopy continuation methods for complementarity problems, 1988. manuscript, IBM Almaden Research Center, San Jose, California. 
[4] M. Kojima and Y. Ye and N. Megiddo. An interior point potential reduction algorithm for the linear complementarity problems. Technical report, IBM Almaden Research Center, San Jose, California, 1988.

[5] M. Kojima, S. Mizuno, and T. Noma. A new continuation method for complementarity problems with uniform $p$-functions. Mathematical Programming, 43:107-113, 1989.

[6] M. Kojima, S. Mizuno, and A. Yoshise. An $O(\sqrt{n} L)$ iteration potential reduction algorithm for linear complementarity problems. Math. Prog., 50:331-342, 1991.

[7] M. Kojima, S. Mizuno, and A. Yoshise. A primal-dual algorithm for a class of linear complementarity problems. Mathematical Programming, 44:1-26, 1989.

[8] M. Kojima, S. Mizuno, and A. Yoshise. A primal-dual interior point method for linear programming. In Nimrod Megiddo, editor, Progress in Mathematical programming, interior-point and related methods, pages 29-47. Springer-Verlag, New York, 1989.

[9] I.J. Lustig, R.E. Marsten, and D.F. Shanno. Computational experience with a primal-dual interior point method for linear programming. Technical Report SOR 89-17, Dept. Civil Eng. and O.R., Princeton University, 1989.

[10] N. Megiddo. Pathways to the optimal set in linear programming. In Nimrod Megiddo, editor, Progress in Mathematical programming, interior-point and related methods, pages 131-158. Springer-Verlag, New York, 1989.

[11] R.C. Monteiro and I. Adler. Interior path-following primal-dual algorithms. Part I: linear programming. Math. Prog., 44:27-41, 1989.

[12] R.C. Monteiro and I. Adler. Interior path-following primal-dual algorithms. Part II: convex quadratic programming. Math. Prog., 44:43-66, 1989. 
[13] M.J. Todd and Yinyu Ye. A centered projective algorithm for linear programming. Technical Report No. 763, School of Operations Research and Industrial Engineering, Cornell University, 1987 (revised 1989). To appear in Math. Prog.

[14] Yinyu Ye. Interior point algorithms for quadratic programming. Technical report, Dept. of Management Sciences, The University of Iowa, 1989. Working Paper Series No. 89-29.

[15] Yinyu Ye. Interior point algorithms for global optimization. Technical report, Dept. of Management Sciences, The University of Iowa, 1990. Working Paper Series No. 90-2.

[16] Yin Zhang, R. A. Tapia, and J. E. Dennis. On the superlinear and quadratic convergence of primal-dual interior point linear programming algorithms. Technical Report TR90-6, Dept. Mathematical Sciences, Rice University, 1990. To appear in SIAM J. on Optimization.

[17] Y. Zhang, R. A. Tapia and F. Potra. On the superlinear convergence of interior point algorithms for a general class of problems. Technical Report TR90-9, Dept. Mathematical Sciences, Rice University, 1990.

[18] Y. Zhang and R. A. Tapia. A quadratically convergent polynomial primal-dual interior point algorithm for linear programming. Technical Report TR90-40, Dept. Mathematical Sciences, Rice University, Houston, Texas 77251-1892, 1991. 